International Journal of Pure and Applied Mathematics

Volume 95 No. $3 \quad 2014,413-426$

ISSN: 1311-8080 (printed version); ISSN: 1314-3395 (on-line version)

url: http://www.ijpam.eu

doi: http://dx.doi.org/10.12732/ijpam.v95i3.9

ijpam.eu

\title{
LAPLACE VARIATIONAL ITERATION METHOD FOR INTEGRO-DIFFERENTIAL EQUATIONS OF FRACTIONAL ORDER
}

\author{
Toheeb A. Biala ${ }^{1}$, Yusuf O. Afolabi ${ }^{2}$, Oladapo O. Asim ${ }^{3}$ \\ ${ }^{1}$ Department of Mathematics \\ University of Ilorin \\ P.M.B. 1515 Ilorin, NIGERIA \\ ${ }^{2}$ Department of Mathematics \\ Sokoto State University \\ P.M.B. 2134 Airport Road, Sokoto, NIGERIA \\ ${ }^{3}$ Department of Mathematics and Physics \\ Osun State University \\ Osogbo, NIGERIA
}

\begin{abstract}
Fractional Integro-Differential Equations (FIDEs) arise in the mathematical modelling of physical phenomena and play an important role in various branches of science and engineering. With He's variational iteration method, it is possible to obtain exact or better approximate solutions of differential equations. This paper is concerned with the solution of FIDEs by the variational iteration method via the Laplace transform. In this approach, a correction functional is constructed by a general Lagrange multiplier, which is determined by using the Laplace transform with the variational theory. The results of applying this method to the studied FIDEs show the high accuracy, simplicity and efficiency of the approach.
\end{abstract}

AMS Subject Classification: 65L03, 45J05

Key Words: variational iteration method, Laplace transform, integro-differential equation, fractional calculus, Lagrange multiplier

Received: April 27, 2014

(C) 2014 Academic Publications, Ltd. url: www.acadpubl.eu 


\section{Introduction}

Fractional integro-differential equations have successfully modelled many physical and engineering phenomena such as heat conduction in material with memory, diffusion processes and so on. In this paper, we consider the important class of FIDE in the fractional initial value problem written in the form

$$
D^{\alpha} y(t)=a(t) y(t)+f(t)+\int_{0}^{t} K(t, x) F(y(x)) d x, \quad y^{(k)}(0)=y_{0}^{(k)}
$$

where $D^{\alpha}$ denotes the fractional differential derivative in the sense of Caputo, $y^{(k)}(t)$ is the $k t h$ derivative of $y$ and $y_{0}^{(k)}$ are the specified initial conditions, $m-1<\alpha \leq m$ and $k=0(1)(m-1)$.

Fractional derivatives and integrals have received considerable interest in recent years though it is a very old subject area. In many applications, fractional derivatives provide more accurate models of systems under consideration. Although the subject of the FIDEs is very old, it does not appear to have been considered systematically in modern literature, and its applicability range is severely limited, due possibly to the difficulty of solving the problems with fractional derivatives and integrals even with numerical simulation. The variational iteration method (VIM) is a strong candidate for approximate analytical solutions to these FIDEs and it involves the identification of a good Lagrange multiplier.

The Lagrange multiplier technique was first proposed by Inokuti et al [9] to solve nonlinear problems which arise in mathematical physics and other related areas. It later developed into a powerful tool (variational iteration method) for solving differential equations. The VIM has been successfully applied on initial value problems, the schrodinger equations, integro-differential equations, fractional differential equations, partial differential equations, q-difference equations. quadratic Riccati differential equations, fractional diffussion wave equations etc [1-4], [6-8], [10-15].

The application of VIM to differential equations usually involve the following steps

1. obtaining the correction functional

2. identifying the Lagrange multiplier

3. determining a good initial approximation

Step 2 and 3 above are to be carefully determined and chosen for a rapid convergence of the solution. Applications of the VIM to fractional differential 
equations mainly and directly use the Lagrange multiplier in ordinary differential equations (ODEs) [1] and this results in slow convergence. Wu and Baleanu [15] noted that the integration by part of the Riemann-Liouville (RL) integral emerging from the constructed correction functional may be difficult to apply. Inorder to overcome this deficiency, they proposed the identification of the general Lagrange multiplier via the Laplace transform.

\section{Basic Definitions}

In this section, we give some definitions and properties of fractional calculus which would be useful in the sequel.

Definition 1. A real function $h(t), t>0$ is said to be in the space $C_{\mu}$, $\mu \in \mathbb{R}$, if there exists a real number $p>\mu$ such that $h(t)=t^{p} h(t)$, where $h_{1}(t) \in C(0, \infty)$ and it is said to be in the space $C_{\mu}^{m}$ if and only if $h^{(m)} \in C_{\mu}$, $m \in \mathbb{N}$.

Definition 2. The fractional derivative $D^{\alpha}$ of $h(t)$ in the Caputo's sense is defined as

$$
D^{\alpha} h(t)=\frac{1}{\Gamma(m-\alpha)} \int_{0}^{t}(t-\tau)^{m-\alpha-1} h^{(m)}(\tau) d \tau
$$

for $m-1<\alpha \leq m, m \in \mathbb{N}, t>0, h \in C_{-1}^{m}$ and $\Gamma(a)$ is the well known Gamma function.

Definition 3. The Riemann-Liouville fractional integral operator $J^{\alpha}$ of order $\alpha>0$, of a function $h \in C_{\mu}, \mu \geq-1$, is defined as

$$
\begin{aligned}
& J^{\alpha} h(t)=\frac{1}{\Gamma(\alpha)} \int_{0}^{t}(t-\tau)^{\alpha-1} h(\tau) d \tau \quad \alpha>0 \\
& J^{0} h(t)=h(t) .
\end{aligned}
$$

Some of the properties of the operator $J^{\alpha}$ are as follows:

(1) $J^{\alpha} J^{\beta} h(t)=J^{\alpha+\beta} h(t)$;

(2) $J^{\alpha} J^{\beta} h(t)=J^{\beta} J^{\alpha} h(t)$;

(3) $J^{\alpha} t^{\gamma}=\frac{\Gamma(\gamma+1)}{\Gamma(\alpha+\gamma+1)} t^{\alpha+\gamma}$. 


\section{Variational Iterartion Method}

\subsection{Basics of the Variation Iteration Method}

In order to illustrate the basic idea of the technique, consider the following general nonlinear integro-differential equation:

$$
\frac{d^{m} y}{d t^{m}}=a(t) y+f(t)+\int_{0}^{t} K(t, x) F(y(x)) d x
$$

where $y=y(t), a(t)$ and $f(t)$ are continuous functions and $\frac{d^{m} y}{d t^{m}}$ is the term of the highest order derivative.

The basic idea of the method is to construct the following correction functional for (2) of the form

$$
y_{n+1}=y_{n}+\int_{0}^{t} \lambda(t, \tau)\left(\frac{d^{m} y}{d t^{m}}-a(t) y(t)-f(t)-\int_{0}^{t} K(t, x) F(y(x)) d x\right)
$$

where $\lambda(t, \tau)$ is called the general Lagrange multiplier [1-4] and $y_{n}$ is the nth order approximate solution.

According to the VIM's rules [1-4], the integration by parts plays an important role in the derivation of the Lagrange multipliers. But in fractional calculus, generally, the following integration by parts cannot hold:

$$
J^{\alpha}\left[v D^{\alpha} u\right]=[u v]_{0}^{t}-J^{\alpha}\left[u D^{\alpha} v\right]
$$

where $v=v(t), D^{\alpha}$ and $J^{\alpha}$ are the notations of the Caputo derivative and the Riemann-Liouville integration, respectively. That's why the VIM was not so successful as other analytical methods such as the Adomian decomposition method (ADM) [23] and the homotopy perturbation method (HPM) [5] in fractional calculus. For this reason, we consider the following reconstruction of the method using the Laplace transform.

\subsection{Laplace Variational Iterative Method (LVIM)}

In the early applications of VIM to fractional differential equations, the term $D^{\alpha} y$ is considered as a restricted varaiation and this results in poor convergence. Following Tsai and Chen [17], Zeng ang Qin [18], and Javidi and Raji [19], Wu and Baleanu [14] proposed the Laplace VIM.

The following Laplace transform [20-22] of the term $D^{\alpha} y(t)$ holds:

$$
L\left[D^{\alpha} y(t)\right]=s^{\alpha} Y(s)-\sum_{k=0}^{m-1} y^{(k)}\left(0^{+}\right) s^{\alpha-k-1}, \quad m-1<\alpha \leq m
$$


Inorder to ilustrate the basic idea of the LVIM for FIDEs, we consider the FIDE (1). The basic steps involved are given as follows:

1. Take the Laplace transform of (1), then the correction functional is

$$
Y_{n+1}(s)=Y_{n}(s)+\lambda(s)\left(s^{\alpha} Y_{n}(s)-\sum_{k=0}^{m-1} y^{(k)}\left(0^{+}\right) s^{\alpha-k-1}-L\left(h_{n}(t)\right)\right)
$$

where $h_{n}(t)=a(t) y_{n}(t)+f(t)+\int_{0}^{t} K(t, x) F\left(y_{n}(x)\right) d x$

2. Regarding the terms $a(t) y_{n}(t)$ and $\int_{0}^{t} K(t, x) F\left(y_{n}(x)\right) d x$ as restricted variations, we make (6) stationary with respect to $Y_{n}$

$$
\delta Y_{n+1}(s)=\delta Y_{n}(s)+\lambda(s)\left(s^{\alpha} \delta Y_{n}(s)\right)
$$

From (7), we determine the Lagrange multiplier as

$$
\lambda(s)=-\frac{1}{s^{\alpha}}
$$

3. The successive approximations are obtained by taking the inverse Laplace transform to obtain

$$
\begin{aligned}
y_{n+1}(t) & =y_{n}(t)-L^{-1}\left[\frac{1}{s^{\alpha}}\left(s^{\alpha} Y_{n}(s)-\sum_{k=0}^{m-1} y^{(k)}\left(0^{+}\right) s^{\alpha-k-1}-L\left[h_{n}(t)\right]\right)\right] \\
& =L^{-1}\left[\frac{1}{s^{\alpha}}\left(\sum_{k=0}^{m-1} y^{(k)}\left(0^{+}\right) s^{\alpha-k-1}+L\left[h_{n}(t)\right]\right)\right]
\end{aligned}
$$

with initial approximation

$$
y_{0}(t)=L^{-1}\left(\sum_{k=0}^{m-1} y^{(k)}\left(0^{+}\right) s^{-k-1}\right)
$$

We give a Lemma below which will be useful in the course of our application

Lemma 3.1. If $F(s)$ is the Laplace transform of $f(t)$, then

$$
L^{-1}\left[\frac{F(s)}{s^{\alpha}}\right]=\frac{1}{\Gamma(\alpha)} \int_{0}^{t}(t-\tau)^{\alpha-1} f(\tau) d \tau
$$


Proof. Let $h(t)=J^{\alpha} f(t)$, then $D^{\alpha} h(t)=f(t)$ so that

$$
L[f(t)]=L\left[D^{\alpha} h(t)\right]=s^{\alpha} L[h(t)]-\sum_{k=0}^{m-1} h^{(k)}\left(0^{+}\right) s^{\alpha-k-1} \quad m-1<\alpha \leq m
$$

Therefore, $L[f(t)]=s^{\alpha} L[h(t)]$

$\Rightarrow$

$$
\begin{gathered}
h(t)=L^{-1}\left[\frac{F(s)}{s^{\alpha}}\right] \\
L^{-1\left[\frac{F(s)}{s^{\alpha}}\right]}=J^{\alpha}[f(t)] \\
=\frac{1}{\Gamma(\alpha)} \int_{0}^{t}(t-\tau)^{\alpha-1} f(\tau) d \tau
\end{gathered}
$$

\section{Applications}

In this section, we apply the above procedure to some FIDEs to obtain exact/approximate solutions.

Example 4.1. We consider the FIDE

$$
y^{0.75}(t)=\left(\frac{-t^{2} e^{t}}{5}\right) y(t)+\frac{6 t^{2.25}}{\Gamma(3.25)}+\int_{0}^{t} e^{t} x y(x) d x, y(0)=0
$$

The analytic solution is given by

$$
\text { Exact }: y(t)=t^{3}
$$

Taking the Laplace transform of both sides, we have the iteration formula

$$
\begin{aligned}
& Y_{n+1}(s)=Y_{n}(s)+ \\
& \lambda(s)\left[s^{0.75} Y_{n}(s)-s^{0.75-1} y(0)-L\left(\left(\frac{-t^{2} e^{t}}{5}\right) y_{n}(t)+\frac{6 t^{2.25}}{\Gamma(3.25)}+\int_{0}^{t} e^{t} x y_{n}(x) d x\right)\right]
\end{aligned}
$$

with the Lagrange multiplier 


$$
\lambda(s)=-\frac{1}{s^{0.75}}
$$

Taking the inverse Laplace transform, we obtain

$$
\begin{aligned}
y_{n+1}(t)=y_{n}(t) & -L^{-1}\left\{\frac { 1 } { s ^ { 0 . 7 5 } } \left[s^{0.75} Y_{n}(s)-L\left(\left(\frac{-t^{2} e^{t}}{5}\right) y_{n}(t)+\frac{6 t^{2.25}}{\Gamma(3.25)}\right.\right.\right. \\
& \left.\left.\left.+\int_{0}^{t} e^{t} x y_{n}(x) d x\right)\right]\right\} \\
= & L^{-1}\left\{\frac{1}{s^{0.75}}\left[L\left(\left(\frac{-t^{2} e^{t}}{5}\right) y_{n}(t)+\frac{6 t^{2.25}}{\Gamma(3.25)}+\int_{0}^{t} e^{t} x y_{n}(x) d x\right)\right]\right\}
\end{aligned}
$$

with initial iteration $y_{0}(t)=0$. Therefore,

$$
\begin{aligned}
y_{1}(t) & =L^{-1}\left\{\frac{1}{s^{0.75}} L\left[\frac{6 t^{2.25}}{\Gamma(3.25)}\right]\right\} \\
& =L^{-1}\left\{\frac{1}{s^{0.75}}\left[\frac{6 \Gamma(3.25)}{s^{3.25} \Gamma(3.25)}\right]\right\}
\end{aligned}
$$

and from the lemma of the previous section, we obtained $y_{1}(t)=t^{3}$

$$
\begin{aligned}
y_{2}(t) & =L^{-1}\left\{\frac{1}{s^{0.75}} L\left[\left(\left(\frac{-t^{2} e^{t}}{5}\right) y_{1}(t)+\frac{6 t^{2.25}}{\Gamma(3.25)}+\int_{0}^{t} e^{t} x y_{1}(x) d x\right)\right]\right\} \\
& =L^{-1}\left\{\frac{1}{s^{0.75}} L\left[\frac{-t^{5} e^{t}}{5}+\frac{6 t^{2.25}}{\Gamma(3.25)}+\int_{0}^{t} e^{t} x^{4} d x\right]\right\} \\
& =L^{-1}\left\{\frac{1}{s^{0.75}} L\left[\frac{-t^{5} e^{t}}{5}+\frac{6 t^{2.25}}{\Gamma(3.25)}+\frac{t^{5} e^{t}}{5}\right]\right\} \\
& =t^{3}
\end{aligned}
$$

Thus, the exact solution $y(t)=t^{3}$ is obtained.

Example 4.2. As a second example, we consider the nonlinear FIDE

$$
\begin{gathered}
D^{\alpha} y(t)=1+\int_{0}^{t} e^{-x} y^{2}(x) d x, \quad 0<\alpha \leq 1 \\
y(0)=1
\end{gathered}
$$

Exact: The exact solution is only known for $\alpha=1$ and is $y(t)=e^{t}$ 
Apllying the Laplace transform to both sides of the FIDE, we obtain

$$
Y_{n+1}(s)=Y_{n}(s)+\lambda(s)\left(s^{\alpha} Y_{n}(s)-s^{\alpha-1} y(0)-L\left[1+\int_{0}^{t} e^{-x} y_{n}^{2}(x) d x\right]\right)
$$

The Lagange multiplier is

$$
\lambda(s)=-\frac{1}{s^{\alpha}}
$$

Taking the inverse Laplace transform, we obtain

$$
y_{n+1}(t)=L^{-1}\left[\frac{y(0)}{s}\right]+L^{-1}\left(\frac{1}{s^{\alpha}} L\left[1+\int_{0}^{t} e^{-x} y_{n}^{2}(x) d x\right]\right)
$$

with initial approximation

$$
\begin{gathered}
y_{0}(t)=1 . \\
y_{1}(t)=1+L^{-1}\left(\frac{1}{s^{\alpha}} L\left[1+\int_{0}^{t} e^{-x} d x\right]\right) \\
=1+L^{-1}\left(\frac{1}{s^{\alpha}} L\left[2-e^{-t}\right]\right) \\
=1+\frac{1}{\Gamma(\alpha)} \int_{0}^{t}(t-\tau)^{\alpha-1}\left(2-e^{-\tau}\right) d \tau
\end{gathered}
$$

To simplify the integration, we use the truncated series representation of $e^{-t}$ (i.e $e^{-t} \approx 1-t+\frac{t^{2}}{2}-\frac{t^{3}}{6}$ )

$$
\begin{aligned}
y_{1}(t) & =1+\frac{1}{\Gamma(\alpha)} \int_{0}^{t}(t-\tau)^{\alpha-1}\left(1+\tau-\frac{\tau^{2}}{2}+\frac{\tau^{3}}{6}\right) d \tau \\
& =1+\frac{t^{\alpha}}{\Gamma(\alpha+1)}+\frac{t^{\alpha+1}}{\Gamma(\alpha+2)}-\frac{t^{\alpha+2}}{\Gamma(\alpha+3)}+\frac{t^{\alpha+3}}{\Gamma(\alpha+4)} \\
& =1+C_{0} t^{\alpha}+C_{1} t^{\alpha+1}-C_{2} t^{\alpha+2}+C_{3} t^{\alpha+3}
\end{aligned}
$$

where $C_{0}=\frac{1}{\Gamma(\alpha+1)}, C_{1}=\frac{1}{\Gamma(\alpha+2)}, C_{2}=\frac{1}{\Gamma(\alpha+3)}, C_{3}=\frac{1}{\Gamma(\alpha+4)}$

$$
y_{2}(t)=1+L^{-1}\left(\frac{1}{s^{\alpha}} L\left[1+\int_{0}^{t} e^{-x} y_{1}^{2}(x) d x\right]\right)
$$




$$
\begin{aligned}
=1+ & L^{-1}\left(\frac { 1 } { s ^ { \alpha } } L \left[1+\int_{0}^{t}\left(1-x+\frac{x^{2}}{2}-\frac{x^{3}}{6}\right)\left(1+C_{0} x^{\alpha}+C_{1} x^{\alpha+1}\right.\right.\right. \\
& \left.\left.\left.-C_{2} x^{\alpha+2}+C_{3} x^{\alpha+3}\right)^{2} d x\right]\right) \\
=1 & \frac{1}{\Gamma(\alpha)} \int_{0}^{t}(t-\tau)^{\alpha-1}\left[1+\int_{0}^{\tau}\left(1-x+\frac{x^{2}}{2}-\frac{x^{3}}{6}\right)\left(1+C_{0} x^{\alpha}\right.\right. \\
& \left.\left.+C_{1} x^{\alpha+1}-C_{2} x^{\alpha+2}+C_{3} x^{\alpha+3}\right)^{2} d x\right] d \tau \\
=1 & +\frac{t^{\alpha}}{\Gamma(\alpha+1)}+\frac{t^{\alpha+1}}{\Gamma(\alpha+2)}-\frac{t^{\alpha+2}}{\Gamma(\alpha+3)}+\frac{t^{\alpha+3}}{\Gamma(\alpha+4)}-\frac{t^{\alpha+4}}{\Gamma(\alpha+5)}+\cdots
\end{aligned}
$$

where we have decided to write the first few terms of $y_{2}(t)$.

Table 1: Computing Exact Errors with $\alpha=1$ for Example 4.2

\begin{tabular}{cccc}
\hline$t$ & Theoretical solution & Numerical solution & Exact error \\
\hline 0.0 & 1.0000000000 & 1.0000000000 & 0 \\
0.1 & 1.1051709181 & 1.11051784324 & $7.51431 \times 10^{-6}$ \\
0.2 & 1.2214027582 & 1.2215102828 & $1.07525 \times 10^{-4}$ \\
0.3 & 1.3498588076 & 1.3503404619 & $4.81654 \times 10^{-4}$ \\
0.4 & 1.4918246976 & 1.4931528164 & $1.32812 \times 10^{-3}$ \\
0.5 & 1.6487212707 & 1.6514967614 & $2.77549 \times 10^{-3}$ \\
0.6 & 1.8221188004 & 1.8269146112 & $4.79581 \times 10^{-3}$ \\
0.7 & 2.0137527075 & 2.0208658118 & $7.11310 \times 10^{-3}$ \\
0.8 & 2.2255409285 & 2.2346437883 & $9.10286 \times 10^{-3}$ \\
0.9 & 2.4596031111 & 2.4692805474 & $9.67744 \times 10^{-3}$ \\
1.0 & 2.7182818285 & $2 / 7254336135$ & $7.15178 \times 10^{-3}$ \\
\hline
\end{tabular}

Example 4.3. Finally, we consider the FIDE

$$
\begin{gathered}
D^{\alpha} y(t)=t\left(1+e^{t}\right)+3 e^{t}+y(t)-\int_{0}^{t} y(x) d x, \quad 3<\alpha \leq 4 \\
y(0)=1, y^{\prime}(0)=1, y^{\prime \prime}(0)=2, y^{\prime \prime \prime}(0)=3
\end{gathered}
$$

Exact: The exact solution is only known for $\alpha=4$ and is $y(t)=1+t e^{t}$

The iterartion formula, after applying the Laplace transform to both sides of the FIDE, is given as 
Table 2: Approximate solution for some $\alpha$ for Example 4.2

\begin{tabular}{cccc}
\hline$t$ & $\alpha=0.25$ & $\alpha=0.50$ & $\alpha=0.75$ \\
\hline 0.0 & 1.0000000000 & 1.0000000000 & 1.0000000000 \\
0.1 & 1.7230900547 & 1.3897504958 & 1.2060055847 \\
0.2 & 2.0082595405 & 1.6088804730 & 1.3708589573 \\
0.3 & 2.2930587249 & 1.8253012680 & 1.5395956852 \\
0.4 & 2.5878218076 & 2.0528287190 & 1.7197612590 \\
0.5 & 2.8927931885 & 2.2957382673 & 1.9150557172 \\
0.6 & 3.2059205726 & 2.5552515792 & 2.1276966297 \\
0.7 & 3.5244125745 & 2.8311288065 & 2.3590254999 \\
0.8 & 3.8451519219 & 3.1222114723 & 2.6096999253 \\
0.9 & 4.1647785103 & 3.4266206079 & 2.8797367780 \\
1.0 & 4.4796441424 & 3.7418035006 & 3.1684807209 \\
\hline
\end{tabular}

$$
\begin{aligned}
Y_{n+1}(s)=Y_{n}(s)+\lambda(s) & \left(s^{\alpha} Y_{n}(s)-\sum_{k=0}^{3} y^{(k)}\left(0^{+}\right) s^{\alpha-k-1}\right. \\
& \left.-L\left[t+t e^{t}+3 e^{t}+y_{n}(t)-\int_{0}^{t} y_{n}(x) d x\right]\right)
\end{aligned}
$$

with Lagrange multiplier

$$
\lambda(s)=-\frac{1}{s^{\alpha}}
$$

Taking the inverse Laplace transform, we obtain

$$
\begin{aligned}
y_{n+1}(t)=L^{-1} & {\left[\frac{y(0)}{s}+\frac{y^{\prime}(0)}{s^{2}}+\frac{y^{\prime \prime}(0)}{s^{3}}+\frac{y^{\prime \prime \prime}(0)}{s^{4}}\right] } \\
& +L^{-1}\left\{\frac{1}{s^{\alpha}} L\left[t+t e^{t}+3 e^{t}+y_{n}(t)-\int_{0}^{t} y_{n}(x) d x\right]\right\} \\
=1 & +t+t^{2}+\frac{t^{3}}{2}+L^{-1}\left\{\frac{1}{s^{\alpha}} L\left[t+t e^{t}+3 e^{t}+y_{n}(t)-\int_{0}^{t} y_{n}(x) d x\right]\right\}
\end{aligned}
$$

with initial approxiamtion

$$
y_{0}(t)=1+t+t^{2}+\frac{t^{3}}{2} .
$$




$$
\begin{aligned}
y_{1}(t)=1+ & t+t^{2}+\frac{t^{3}}{2}+L^{-1}\left\{\frac { 1 } { s ^ { \alpha } } L \left[t+t e^{t}+3 e^{t}+1+t+t^{2}+\frac{t^{3}}{2}\right.\right. \\
& \left.\left.\quad-\int_{0}^{t}\left(1+x+x^{2}+\frac{x^{3}}{2}\right) d x\right]\right\} \\
= & 1+t+t^{2}+\frac{t^{3}}{2}+L^{-1}\left\{\frac{1}{s^{\alpha}} L\left[1+t+\frac{t^{2}}{2}+\frac{t^{3}}{6}-\frac{t^{4}}{8}+(3+t) e^{t}\right]\right\}
\end{aligned}
$$

To simplify the integration, we took the truncated Taylor's expansion of the exponential term i.e $e^{t} \approx 1+t+\frac{t^{2}}{2}+\frac{t^{3}}{6}$ and obtained, after applying the inverse Laplace transform,

$y_{1}(t)=1+t+t^{2}+\frac{t^{3}}{2}+\frac{4 t^{\alpha}}{\Gamma(\alpha+1)}+\frac{5 t^{\alpha+1}}{\Gamma(\alpha+1)}+\frac{6 t^{\alpha+2}}{\Gamma(\alpha+3)}+\frac{7 t^{\alpha+3}}{\Gamma(\alpha+4)}+\frac{t^{\alpha+4}}{\Gamma(\alpha+5)}$

Table 3: Computing Exact Errors with $\alpha=4$ for Example 4.3

\begin{tabular}{cccc}
\hline$t$ & Theoretical solution & Numerical solution & Exact error \\
\hline 0.0 & 1.0000000000 & 1.0000000000 & 0 \\
0.1 & 1.1105170918 & 1.1105170918 & $1.76104 \times 10^{-12}$ \\
0.2 & 1.2442805516 & 1.2442805512 & $4.57431 \times 10^{-10}$ \\
0.3 & 1.4049576422 & 1.4049576304 & $1.18956 \times 10^{-8}$ \\
0.4 & 1.5967298879 & 1.5967297585 & $1.2058 \times 10^{-7}$ \\
0.5 & 1.8243606354 & 1.8243599059 & $7.29441 \times 10^{-7}$ \\
0.6 & 2.0932712802 & 2.0932680966 & $3.18366 \times 10^{-6}$ \\
0.7 & 2.4096268952 & 2.4096158024 & $1.10928 \times 10^{-5}$ \\
0.8 & 2.7804327428 & 2.7803999655 & $3.27773 \times 10^{-5}$ \\
0.9 & 3.2136280004 & 3.2135574025 & $8.53975 \times 10^{-5}$ \\
1.0 & 3.7182818285 & 3.7180803571 & $2.01471 \times 10^{-4}$ \\
\hline
\end{tabular}

\section{Conclusion}

The Laplace-Lagrange multiplier is proposed from the Laplace transform and incorporated with the techniques of VIM to produce exact/approximate solutions to integro-differential equations of fractional order. With this approach, 
Table 4: Approximate solution for some $\alpha$ for Example 4.3

\begin{tabular}{cccc}
\hline$t$ & $\alpha=3.25$ & $\alpha=3.50$ & $\alpha=3.75$ \\
\hline 0.0 & 1.0000000000 & 1.0000000000 & 1.0000000000 \\
0.1 & 1.1107796668 & 1.1106118344 & 1.1105440383 \\
0.2 & 1.2467420596 & 1.2453017691 & 1.2446086794 \\
0.3 & 1.4140602225 & 1.4090386155 & 1.4063615817 \\
0.4 & 1.6197481345 & 1.6076109271 & 1.6006532891 \\
0.5 & 1.8716437745 & 1.8476194773 & 1.8330507143 \\
0.6 & 2.1784510795 & 2.1365182585 & 2.1098953690 \\
0.7 & 2.5498077438 & 2.4826796720 & 2.4383774878 \\
0.8 & 2.9963699286 & 2.8954767556 & 2.8266234527 \\
0.9 & 3.5299104233 & 3.3853796176 & 3.2837956583 \\
1.0 & 4.1634287060 & 3.9640648418 & 3.38202046481 \\
\hline
\end{tabular}

the Lagrange multipliers can be determined easily and new variational iteration formulae can be derived. The approach is implemented on some FIDEs to show its simplicity, accuracy and efficiency.

\section{References}

[1] J. H. He, Approximate analytical solution for seepage flow with fractional derivatives in porous media, Computer Methods in Applied Mechanics and Engineering 167 (1-2) (1998) 57-68. doi:10.1016/S0045-7825(98)00108-X.

[2] J. H. He, Variational iteration method- a kind of nonlinear analytical techniques: some examples, International Journal of Nonlinear Mechanics 34 (4) (1999) 699-708. doi:10.1016/S0020-7462(98)00048-L.

[3] J. H. He, Variational iteration method- some recent results and new interpretations, Journal of Computational and Applied Mathematics 207 (1) (2007) 3-17. doi:10.1016/j.cam.2006.07.009.

[4] J. H. He and X. H. Wu, Variational iteration method- new development and applications, Comp. Appl. Math 54 (7-8) (2007) 881-894. doi:10.1016/j.camwa.2006.12.083. 
[5] S. Momani and Z. Odibat, Homotopy perturbation method for nonlinear partial differential equations of fractional order. Phys. Lett. A 365(56),(2007) 345-350. doi:10.1016/j.physleta.2007.01.046.

[6] S. Momani and Z. Odibat, Numerical comparison of methods for solving linear differential equations of fractional order, Chaos, Solitons and Fractals 31 (5) (2007) 1248-1255. doi:10.1016/j.chaos.2005.10.068.

[7] Z. M. Odibat and S. Mamani, Appications of variational iteration method to Nonlinear differential equations of fractional order, International Journal of Nonlinear Science and Numerical simulation, 7 (1) (2006) 27-34. doi.10.1515/IJNSNS.2006.7.1.27.

[8] S. Abbasbandy, A new application of He's variational iteration method for quadratic Riccati differential equation by using Adomian's polynomials, J. Comp. Appl. Math. 207 (1) (2007) 59-63. doi.10.1016/j.cam.2006.07.012.

[9] M. Inokuti, H. Sekine, T. Mura, General use of the Lagrange multiplier in nonlinear mathematical physics. In: Nemat-Nasser, S (ed.) Variational Methods in the Mechanics of Solids, 156-162. Pregman Press, New York (1978).

[10] M. A. Noor and S. T. Mohyu-Din, Variational iteration method for solving higher-order nonlinear boundary value problems using He's polynomials, Int. J. Nonlinear Sci and Numer. Simul. 9 (2) (2008) 141-156. doi.10.1515/IJNSNS.2008.9.2.141.

[11] G. C. Wu, New trends in variational iteration method, Commun. Fract. Calc. 2 (2) (2011), 59 - 75.

[12] G. C. Wu, Variational iteration method for q-difference equations of second order, J. Appl. Math. (2012) Article ID:102850. http://dx.doi.org/10.1155/2012/102850.

[13] G. C. Wu and K. T. Wu, Variational approach for fractional diffusion-wave equation on Cantor sets, Chin Phys. Lett. 29 (6) (2012) Article ID:060505.

[14] G. C. Wu and D. Baleanu, New applications of variational iteration method- from differential equations to q-fractional difference equations, Advances in Difference Equations 2013, 2013. 21 doi:10.1186/1687-1847-201321. 
[15] G. C. Wu and D. Baleanu, Variational iteration method for fractional calculus- a universal approach by Laplace transform, Advances in Difference Equations 2013, 2013. 18 doi:10.1186/1687-1847-2013-18.

[16] A. M. Wazwaz, The variational iteration method for analytic treatment for linear and nonlinear ODEs, Appl. Math. Comp. 212 (1) (2009) 120-134. doi:10.1016/j.amc.2009.02.003.

[17] P. Y. Tsai and C. K. Chen, An approximate analytic solution of the nonlinear Riccati differential equation, J. Franklin Inst. 347 (10) (2010) 18501862. doi:10.1016/j.jfranklin.2010.10.005.

[18] D. Q. and Y. M. Qin, The Laplace-Adomian-Pade technique for the seepage flow with the Riemann-Liouville derivative,Commun. Fract. Calc. 3 (1) (2012) 26-29.

[19] M. Javidi andd M. A. Raji, Combination of Laplace transform and homotopy perturbation method to solve the parabolic partial differential equations, Commun. Fract. Calc. 3(1) (2012) 10-19.

[20] K. B. Oldham and J. Spanier, The Fractional Calculus, Academic Press, New York (1974).

[21] I. Podlubny, Fractional Differential Equations, Academic Press, San Diego (1999).

[22] A. A. Kilbas, H. M. Srvastav and J. J. Trujillo, Theory and Applications of Fractional Differential Equations, Elsevier, New York (2006).

[23] J. S. Duan, R. Rach, D. Baleanu, A. M. Wazwaz, A review of the Adomian decomposition method and its applications to fractional differential equations. Commun. Fract. Calc. 3(2), (2012) 73-99. 